\title{
Tocotrienol-Rich Fraction Of Palm Oil Attenuates Type II Collagen-Induced Temporomandibular Joint Rheumatoid Arthritis In Rats For Future Clinical Application
}

\author{
Ahmed S. Ahmed, \\ Anatomy and embryology department, College of medicine, Tanta \\ university. Tanta, Egypt
}

Doi:10.19044/esj.2020.v16n24p124 URL:http://dx.doi.org/10.19044/esj.2020.v16n24p124

\begin{abstract}
Temporomandibular joint involvement in rheumatoid arthritis (RA) is not uncommon. Nonsteroidal anti-inflammatory drugs (NSAIDs), are considered main line of RA treatment but they can cause side effects. Tocotrienol rich fraction (TRF) extracted from palm oil was reported to possess potent antioxidant and anti-inflammatory activities. This research was designed to study the therapeutic potential of TRF against TMJ arthritis. 30 Albino Wistar rats were divided into: Control (C), Arthritis (A) and Arthritis + TRF (A-TRF) groups. A-TRF group received TRF daily from day 30 up to day 45 after TMJ arthritis induction was accomplished. Serum inflammatory cytokines were assessed and TMJ histopathological examinations were done. Plasma levels of inflammatory cytokines increased in A-group and they were comparable in both $\mathrm{C}$ and A-TRF groups. A-TRF group showed that, the inflammatory changes were mild on examination of stained sections of TMJ. A-TRF-group bone mineral density was notably increased after TRF administration. In conclusion, the decreased plasma levels of inflammatory cytokines and the increased bone mineral density associated with tocotrienolsrich-fraction supplements administration could be provide an evidence that TRF could be used as an effective therapy of TMJ rheumatoid arthritis.
\end{abstract}

Keywords: Tocotrienols, TMJ, Collagen, Rheumatoid, Arthritis

\section{Introduction}

Rheumatoid arthritis (RA) is an autoimmune connective tissue disorder characterized by articular (affects mainly the peripheral joints) and extra articular manifestations (Scott, 2010). About 50\% of RA patients clinically exhibit temporomandibular joint (TMJ) involvement in the form of joint pain, crepitation, swelling and morning stiffness that last more than 30 
minutes (Savtekin, 2018). Due to the effect of inflammatory mediators, the main morphologic changes findings are cartilaginous destruction (with inflammatory cells infiltration) and bony erosion associated with gradual decrease in joint space. Many research papers had reported that the etiology of RA is related to inflammatory cytokines [Tumor necrosis factor- $\alpha$ (TNF- $\alpha$ ), interleukin-1 alpha (IL-1 $\alpha$ ) and interleukin-1 beta (IL-1 $\beta$ )] which play a key role in the joint inflammation pathway (Chen, 2019). These inflammatory cytokines increase the expression of cyclooxygenase-2 (COX-2) causing extra production of prostaglandin E2 (PG-E2) resulting in more cartilaginous destruction (Samuels, 2018).

The use of the RA drugs is associated with adverse events such as changes in mucous membranes and other symptoms that different from patient to another. About 50\% of the patients with RA complain xerostomia, difficulties in swallowing or phonation (Wang, 2018). Long term usage of nonsteroidal anti-inflammatory drugs (NSAIDs) is considered the main line of treatment of RA. Given that, NSAIDs has many side effects (heartburn, stomach ulcers, rashes and liver \& kidney pathology), a true need for naturally occurring alternative are rising on the horizon (Thakur, 2018).

Vitamin E (Vit. E) was first discovered in 1922. It was classified "E" since vitamins A, B, C, and D had already been categorized (Szymańska, 2017). It was initially isolated from wheat germ oil and named as tocopherol (Tp). Then tocotrienol (Tt) was isolated from Hevea brasiliensis plant in 1966 (Ha, 2011). Vit. E family contains eight isomers, namely tocopherols $(\alpha, \beta, \gamma$, and $\delta$ ) and tocotrienols $(\alpha, \beta, \gamma$, and $\delta$ ) that should be obtained from diet. It exhibits various anti-allergic, anti-cancer, anti-diabetic and anti-inflammatory. It was reported that $\mathrm{Tt}$ possess a more potent anti-inflammatory potential than Tp (Khallouki, 2016).

Vit. $\mathrm{E}$ is also possessing potent anti-oxidant activity, that can ameliorate the free radicles related pathology by decreasing the production of inflammatory cytokines (IC) (Das, 2002). One of the richest naturally occurring sources of $\mathrm{Tt}$ is the crude palm oil (CPO) [also referred to as tocotrienols-rich-fraction (TRF)]. It contains approximately $1 \mathrm{gm} / \mathrm{kg}$ of $\mathrm{Tt}$ and reported to be a therapeutic agent for many autoimmune diseases such as intrinsic asthma (Saw, 2019). This study was designed to investigate the therapeutic potential of palm extracted TRF against collagen induced TMJ arthritis (CITMJA) and its ability to reduce the level of plasma inflammatory mediators.

\section{Material and methods}

Animals:

30 Albino Wistar rats were used (average weight and age were 150 gm and 6 months respectively). Animals were housed individually. Free access to 
food and water was allowed.12 light/dark cycle was kept. By the help of air conditions, the temperature was maintained $25^{\circ} \mathrm{C}$ (in accordance to national and institutional guidelines). This research study was approved by Research and Ethics Committee, Quality Assurance Unit, Faculty of Medicine, Tanta University, Egypt.

\section{Experimental design:}

Rats were divided into three groups ( $n=10)$; (1) Control (C) group, received bilateral intraarticular TMJ injection of $0.05 \mathrm{ml}$, complete freund's adjuvant (CFA) (Santa Cruz Biotechnology, Inc., USA) at day 1, 7 and 14. (2) Arthritis (A) group and (3) Arthritis + TRF (A-TRF) group.

Rats of A and A-TRF groups received saline (2ml) and TRF (40 mg/kg b.w.) (Sime Darby Ptd. Ltd., Malaysia) respectively by oral gavage daily from day 30 up to day 45. TMJ arthritis induction was accomplished at day one for both $\mathrm{A}$ and A-TRF groups by bilateral intra-articular injection of $0.2 \mathrm{mg}$ of collagen (type II) from chicken sternum dissolved in CFA into TMJs (Lugo, 2016). Injection was done under general anesthesia then the hair covering TMJ was removed. The posterior end of zygomatic arch was palpated. The tip of insulin syringe was inserted below this point and directed anteromedially (Fuentes, 2017) . Booster doses were administered at day 7 (booster-1) and day 14 (booster-2) (figure 1). Body weight and head circumference (1 mm in front of external auditory meatus) were assessed on weekly basis using digital scale-Tor Rey LEQ 5/10 (Tor Rey, EQ-4HP, Torrey, Mexico City, Mexico) and measuring tape respectively. At day 45, all rats were sacrificed under general anesthesia. Blood samples were collected then centrifuged at 4000 rpm for 10 minutes and serum was stored at $-80^{\circ} \mathrm{C}$ (Özcan, 2018). TMJs were collected for histopathological examinations

\section{Serum biomarker analysis:}

Serum C-reactive protein (CRP) was determined by the help of ab108827 - C Reactive Protein (PTX1) Rat ELISA Kit. For serum TNF- $\alpha$, Rat TNF alpha ELISA Kit (ab236712) was used. For IL-6 and IL-1 $\beta$, Rat IL-6 ELISA Kit (ab234570) and Rat IL-1 beta ELISA Kit (ab255730) were used respectively. All kits were purchased from Abcam plc., USA. The amounts of IC were determined using a standard curve that was run for each of ELISA (Williams, 2019).

\section{Histopathological examination:}

Hematoxylin and eosin staining was done in accordance to (Feldman, 2014). briefly, fresh TMJs was cut into $1 \mathrm{~cm}^{3}$ cubes immediately after extraction from the rats. It was placed in fixative $10 \%$ paraformaldehyde solution and left for 48 hours then placed in tissue processing cassettes. By help of ascending grades of alcohol, tissue is dehydrated to remove water and formalin traces then immersed in xylene to remove alcohol and facilitate paraffin wax infiltration into the tissue. Cassettes were placed on warm plates 
then tissue was removed and immersed in paraffine blocks. After paraffine solidification, the blocks were cut into $5 \mu \mathrm{m}$ thick sections by using manually operated rotary microtome CUT 4050 (4050F, R) (Microtec Laborgeräte GMBH, Germany). Tissue sections were placed on glass microscope slides, rehydrated, stained with hematoxylin (stains nuclei in blue) for 10 minutes and eosin (stains cytoplasm in red) for 10 seconds. The stained tissue sections were dehydrated again by ascending grades of alcohol for 10 minutes than covered by coverslip. Histopathological examinations were performed by two expert histopathologists blinded to our study.

\section{Bone mineral density (BMD):}

At day 45 (before sacrifice), rats' head computerized tomography (CT) scan was conducted by micro-CT scanner, NeoScan S80 (Neoscan, Inc., Belgium). Rats were placed in scanner under general anesthesia (Adise, 2014). Data obtained were analyzed by NRecon 1275 software (Micro Photonics Inc., USA).

\section{Statistical analysis:}

SPSS software, $25 \mathrm{~V}$. (SPSS Inc., USA) was used for data analysis, data were expressed in mean \pm standard deviation and probability value was considered significant if $<0.05$. Tukey-Kramer test was used for groups comparison.

\section{Results}

Total body weight of A-group showed a significant $(\mathrm{p}<0.05)$ decrease within the first 14 days of study if compared to C-group. While that of A-TRFgroup significantly $(\mathrm{p}<0.05)$ increased steadily within the last 15 days of study if compared to A-group (figure 2).

TMJ edema was assessed by measuring head circumference from day 1 up to day 45 on a weekly basis. A-TRF group showed signs of inflammation up to day 30, after that there was a significant $(p<0.05)$ increase in head circumference of A-group if compared to A-TRF group. The reduction of head circumference of A-TRF group was comparable to that of C-group (figure 3).

Plasma levels of CRP, TNF- $\alpha$, IL- $1 \beta$, and IL-6 at day 45 showed a marked $(\mathrm{p}<0.05)$ increase in A-group if compared with $\mathrm{C}$ and A-TRF- groups. While plasma CRP, TNF- $\alpha$, IL-1 $\beta$, and IL-6 levels in A-TRF- group were comparable to C-group (figure 4 a, b, c, d).

Examination of hematoxylin and eosin stained sections of TMJ showed that, in C-group, no signs of inflammation (hyperplasia, inflammatory cells infiltration), arthritis or tissue destructions. A-group showed marked synovial inflammatory cells infiltration with fibrovascular pannus formation in addition to degeneration and erosion of the cartilage with periarticular inflammation and fibrous adhesion. In A-TRF group, the inflammatory 
changes were mild with an increase in the cartilaginous cells number (figure 5).

By help of NRecon software, mean bone densities of rat TMJ were calculated from CT images. The results from this analysis showed that the bone mineral density of TMJ of A-group were decreased if compared to control or A-TRF-group bone densities, while A-TRF-group showed significant increases in bone mineral density compared with that in A-group $(\mathrm{p}<0.05)$ (figure 6).

\section{Discussion}

RA and osteoarthritis (OA) are the two main types of arthritis that affect TMJ. In RA, inflammatory cells attack joint tissue resulting in sever inflammation, while in OA, wear and tear pathology is age related. The various side effects of NSAIDs used in RA and OA treatment hinder their use and developed a true need for naturally present anti-inflammatory agents that can be used safely with limited side effects. TRF extracted from palm oil may be a promising anti-inflammatory agent. Radhakrishnan et al., (2014) reported that $\gamma$-Tt could be considered as an anti-oxidant and protect joint ultrastructure in rat models while Haleagrahara et al., (2014) reported the anti-inflammatory potential of $\delta$-Tt. Zaida et al., (2014) invitro study using $\gamma$-Tt has shown its ability to reduce arthritis related pathology.

Given that $\gamma$-Tt extracted from palm oil has the ability to decrease IC (TNF- $\alpha$, IL-1 $\beta$, IL-6) (Hu, 2017), to the best of our knowledge, no studies have reported the effect of TRF on TMJ rheumatoid arthritis. The collagen induced arthritis rat model provide similar pathological changes (bone destruction, inflammatory cells infiltration, upregulation of IC) as in human rheumatoid arthritis (Bolon, 2011). Kamada et al. (1997) reported that, this rat model of arthritis could be used in the preclinical studies of RA. About $90 \%$ of rats in collagen induced arthritis model showed joint pathology after 30 days of collagen injection which is similar to that of human RA.

In our study, collage (type II) emulsified with CFA was injected directly into the TMJ (bilaterally) which resulted in sever joint swelling, pain and limitation of movement which caused a change in eating pattern of arthritic group evidenced by the decrease in body weight. While those of TRF treated group regain mobility with a noticeable decrease in the inflammation of TMJ started from day 30 (after treatment started) which resulted in a slight regain of eating pattern and body weight which comes in consistent with Sproston et al., (2018). In our study, the external join edema was evidenced by the increase in the total head circumference. This increase was significant in A-group in compared to C-group. The significant decrease in head circumference reported in TRF treated group could provide a suggestion that TRF could ameliorate some of the RA associated clinical manifestation. 
CRP is considered as effective indicator of the inflammatory state of the join (Kim, 2015). Ballou et al., (1992) reported that plasma level of CRP increases in association with bone and cartilage inflammation. In RA, the inflamed synovial membrane can activate macrophages which release CRP in response (Müller, 2005). The more plasma concentration of CRP is considered an indicator for more joint arthritis. Chen et al., (2018) reported that, in RA IL-6 and IL-1 mediate the resulting inflammatory pathway and join pathological changes. The decrease in plasma CRP observed in our study (in A-TRF group) could be due to inactivation of inflammatory cells (macrophages) which comes in consistent with Bondeson (1996) and Wei et al., (2015). It is important to notice that using CRP as the only indicator for $\mathrm{RA}$, is not $100 \%$ accurate, as CRP plasma level increases in association with either inflammatory or infectious joint affection (Scott, 2010). Chimenti et al., (2015) reported that IL-1 $\beta$ and TNF- $\alpha$ are the most frequently released IC in association with arthritis. Harre et al., (2017) stated that, synovial membrane epithelial cells and macrophages help in the production of TNF- $\alpha$ which plays a crucial role in IL-1 and IL-6 production which result in production of other IC such as Matrix metalloproteinases (MMPs) and prostaglandins which cause joint pain and cartilaginous erosion.

\section{Conclusion}

As a conclusion, the significant decrease of plasma levels of inflammatory cytokines and the increased bone mineral density associated with tocotrienols-rich-fraction supplements could be provide an evidence that TRF could be used as an effective therapy of TMJ rheumatoid arthritis.

\section{Competing interests disclaimer:}

Author has declared that no competing interests exist. The products used for this research are commonly and predominantly use products in our area of research and country. There is absolutely no conflict of interest between the author and producers of the products because we do not intend to use these products as an avenue for any litigation but for the advancement of knowledge. Also, the research was not funded by the producing company rather it was funded by personal efforts of the author. 


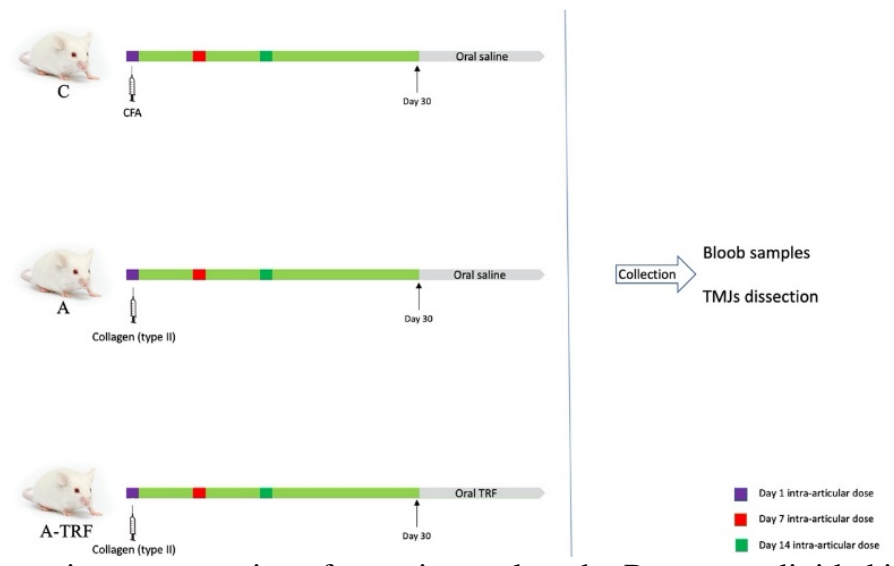

Fig. 1: Schematic representative of experimental study. Rats were divided into three groups (n=10); (1) Control (C) group, (2) Arthritis (A) group and (3) Arthritis + TRF (A-TRF) group. All groups received bilateral TMJ intra-articular injections at days 1, 7 and 14.

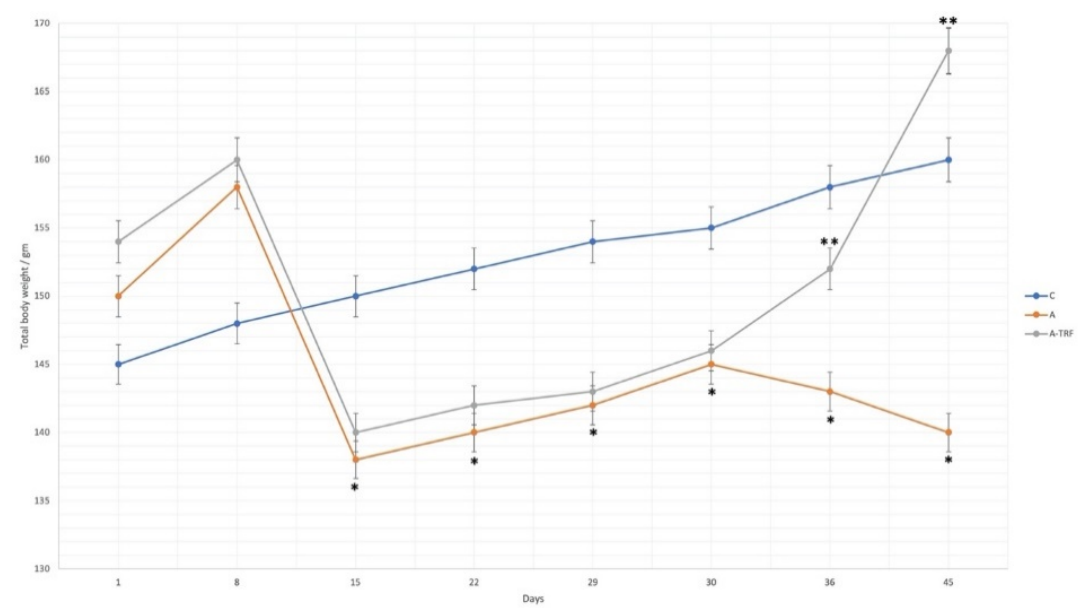

Fig. 2: Effect of tocotrienols-rich-fraction on rats' total body weight. C: Control group. A: Arthritis group. A-TRF: Arthritis + TRF group. $*(\mathrm{p}<0.05)$ Arthritis group in comparison to Control group. ${ }^{* *}(\mathrm{p}<0.05)$ Arthritis + TRF group in comparison to Arthritis group. Data were expressed as means \pm standard deviations. 


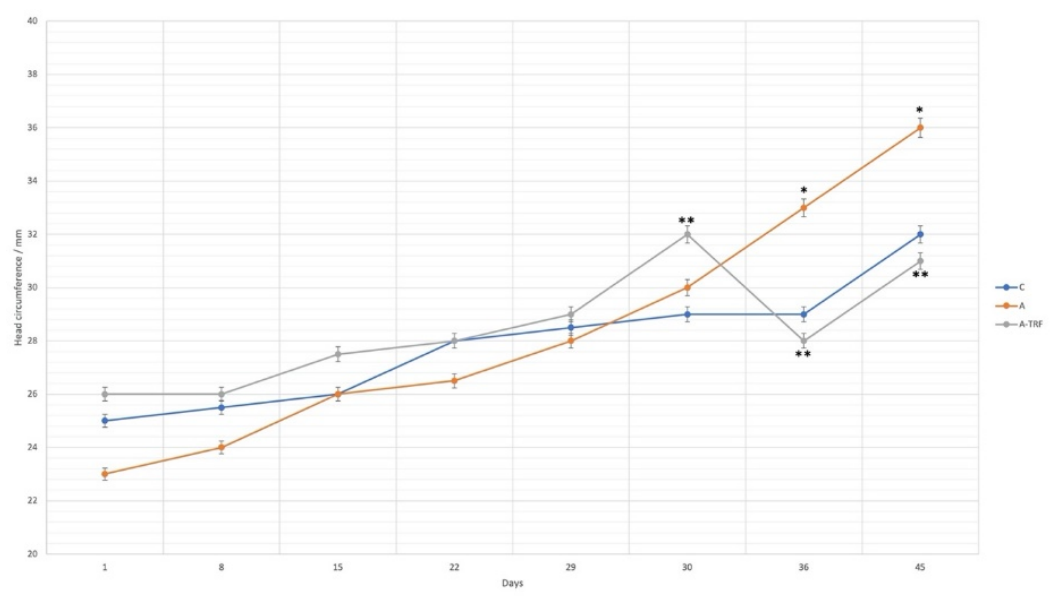

Fig. 3: Effect of tocotrienols-rich-fraction on rats' head circumference. C: Control group. A: Arthritis group. A-TRF: Arthritis + TRF group. * $(\mathrm{p}<0.05)$ Arthritis group in comparison to A-TRF group. ${ }^{* *}(\mathrm{p}<0.05)$ A-TRF group in comparison to control group. Data were expressed as means \pm standard deviations.

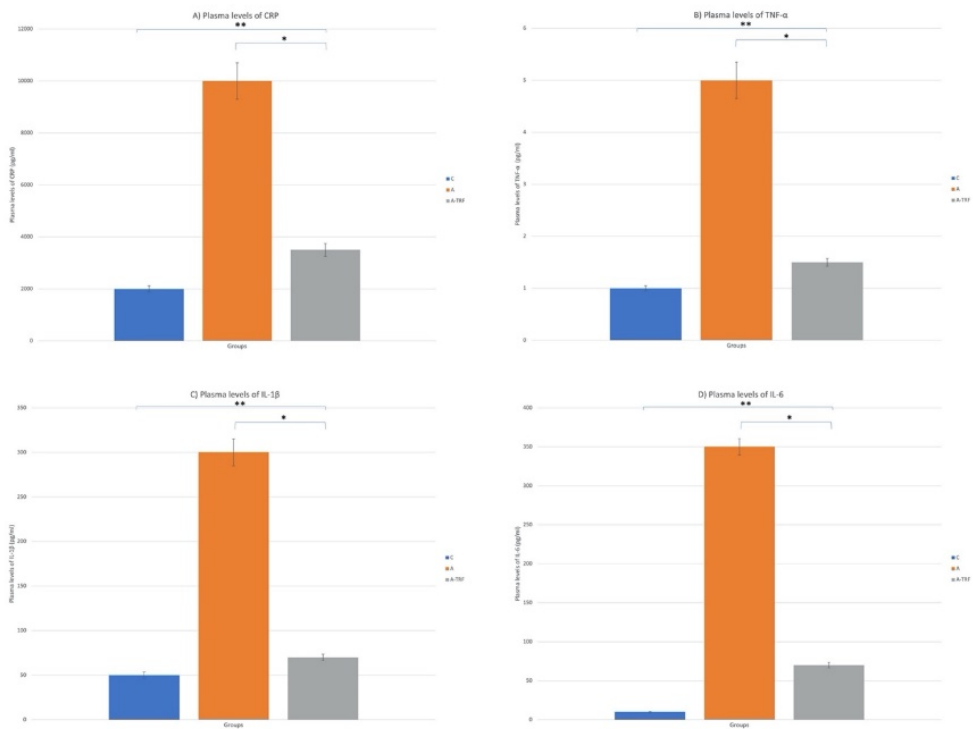

Fig. 4: A) Plasma levels of CRP, B) Plasma levels of TNF- $\alpha$, C) Plasma levels of IL-1 $\beta$, D) Plasma levels of IL-6. All IC show a significant $(p<0.05)$ increase in A-group if compared with C and A-TRF- groups. While plasma CRP, TNF- $\alpha$, IL-1 $\beta$, and IL-6 levels in A-TRFgroup were comparable to C-group * $(\mathrm{p}<0.05)$ Arthritis group in comparison to A-TRF group. ${ }^{* *}(\mathrm{p}<0.05)$ A-TRF group in comparison to control group. Data were expressed as means \pm standard deviations. 

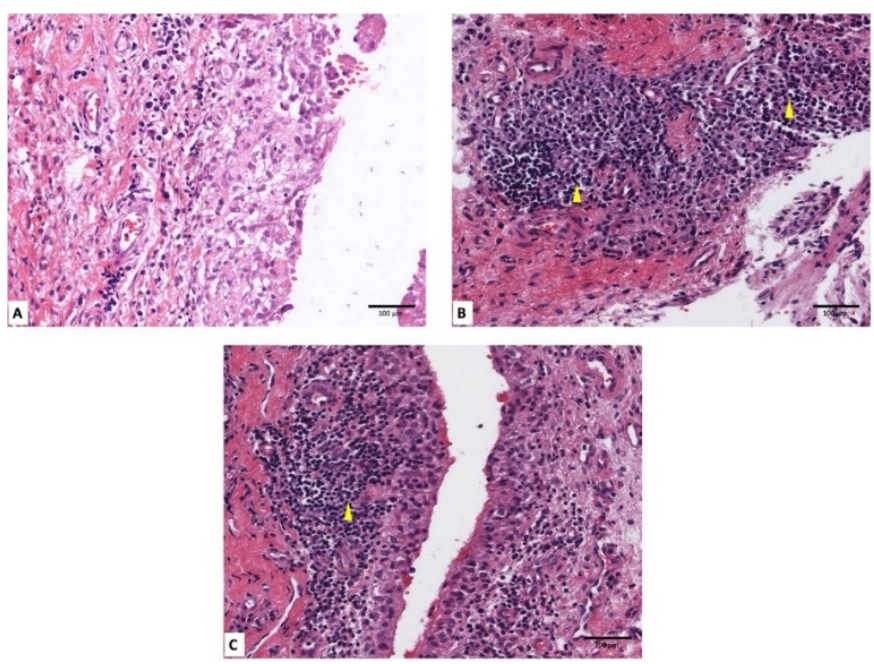

Fig. 5: Photomicrograph of TMJ synovium stained with hematoxylin and eosin (X 1000). yellow arrow, inflammatory cells infiltration. A) C-group, no signs of inflammation (hyperplasia, inflammatory cells infiltration), arthritis or tissue destructions. B) A-group showed marked synovial inflammatory cells infiltration (yellow arrow) with fibrovascular pannus formation. C) A-TRF group, the inflammatory changes were mild if compared to Agroup.

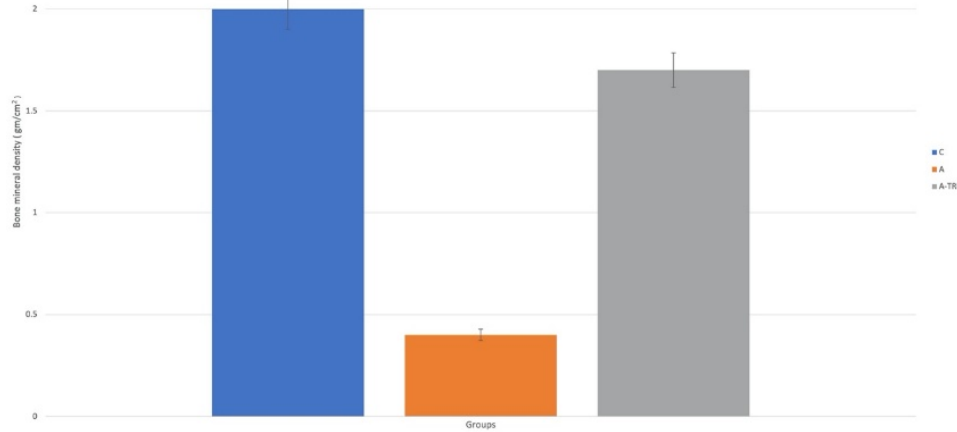

Fig. 6: Effect of tocotrienols-rich-fraction on rats' TMJ bone densities. C: Control group. A: Arthritis group. A-TRF: Arthritis + TRF group. * $(p<0.05)$ BMD of A-group in comparison to A-TRF group. ${ }^{* *}(\mathrm{p}<0.05) \mathrm{BMD}, \mathrm{A}$ - group in comparison to control group. Data were expressed as means \pm standard deviations.

\section{References}

1. Adise S, Saliu A, Maldonado N, Khatri V, Cardoso L, RodríguezContreras A. Effect of maternal care on hearing onset induced by developmental changes in the auditory periphery. J Neurosci. 34(13):4528-4533 (2014). 
2. Ballou, S. P. \& Lozanski, G. Induction of inflammatory cytokine release from cultured human monocytes by C-reactive protein. Cytokine 4, 361-368 (1992).

3. Bolon, B. et al. Rodent preclinical models for developing novel antiarthritic molecules: comparative biology and preferred methods for evaluating efficacy. Journal of biomedicine \& biotechnology 2011, 569068 (2011).

4. Bondeson, J. Effects of tenidap on intracellular signal transduction and the induction of proinflammatory cytokines: a review. General pharmacology 27, 943-956 (1996).

5. Chen Z, Bozec A, Ramming A, Schett G. Anti-inflammatory and immune-regulatory cytokines in rheumatoid arthritis. Nat Rev Rheumatol. 15(1):9-17(2019).

6. Chen, L. et al. Inflammatory responses and inflammation-associated diseases in organs. Oncotarget 9, 7204-7218 (2018)

7. Chimenti, M. S. et al. The interplay between inflammation and metabolism in rheumatoid arthritis. Cell Death \&Amp; Disease 6, e1887 (2015).

8. Das U. A radical approach to cancer. Med Sci Monit. 8(4):RA79RA92 (2002).

9. Feldman AT, Wolfe D. Tissue processing and hematoxylin and eosin staining. Methods Mol Biol. 1180:31-43 (2014).

10. Fuentes R, Veuthey C, Arias A, Saravia D, Ottone NE. Injection in temporomandibular joint of rats. Description of technical protocol. Pol J Vet Sci. 20(2):207-211 (2017).

11. Ha H, Lee JH, Kim HN, Lee ZH. $\alpha$-Tocotrienol inhibits osteoclastic bone resorption by suppressing RANKL expression and signaling and bone resorbing activity. Biochem Biophys Res Commun. 406(4):546551 (2011).

12. Haleagrahara, N., Swaminathan, M., Chakravarthi, S. \& Radhakrishnan, A. Therapeutic efficacy of vitamin E delta-tocotrienol in collagen-induced rat model of arthritis. BioMed research international 2014, 539540 (2014).

13. Harre, U. \& Schett, G. Cellular and molecular pathways of structural damage in rheumatoid arthritis. Seminars in immunopathology 39, 355-363 (2017).

14. Hu, Y., Yang, Y. \& Luo, B. Evaluation of destruction in a collageninduced arthritis rat model: Bony spur formation. Experimental and therapeutic medicine 14, 2563-2567 (2017).

15. Kamada, H., Goto, M., Matsuura, S., Takaoka, Y. \& Nagai, H. Immunopharmacological studies on collagen-induced arthritis in dark 
Agouti (DA) rats. Japanese journal of pharmacology 74, 313-322 (1997).

16. Khallouki F, de Medina P, Caze-Subra S, et al. Molecular and Biochemical Analysis of the Estrogenic and Proliferative Properties of Vitamin E Compounds. Front Oncol. 5:287 (2016).

17. Kim, K. W., Kim, B. M., Moon, H. W., Lee, S. H. \& Kim, H. R. Role of C-reactive protein in osteoclastogenesis in rheumatoid arthritis. Arthritis research \& therapy 17, 41 (2015).

18. Lugo JP, Saiyed ZM, Lane NE. Efficacy and tolerability of an undenatured type II collagen supplement in modulating knee osteoarthritis symptoms: a multicenter randomized, double-blind, placebo-controlled study. Nutr J. 15:14 (2016).

19. Müller-Ladner, U., Pap, T., Gay, R. E., Neidhart, M. \& Gay, S. Mechanisms of disease: the molecular and cellular basis of joint destruction in rheumatoid arthritis. Nature Reviews Rheumatology 1, 102 (2005).

20. Özcan O, Erdal H, İlhan G, et al. Plasma Ischemia-Modified Albumin Levels and Dynamic Thiol/Disulfide Balance in Sickle Cell Disease: A Case-Control Study. Orak Hücre Hastalığında Plazma İskemi Modifiye Albümin Düzeyleri ve Dinamik Tiyol/Disülfit Dengesi: Bir Olgu Kontrol Çalışması. Turk J Haematol. 35(4):265-270 (2018).

21. Radhakrishnan, A., Tudawe, D., Chakravarthi, S., Chiew, G. S. \& Haleagrahara, N. Effect of gamma-tocotrienol in counteracting oxidative stress and joint damage in collagen-induced arthritis in rats. Experimental and therapeutic medicine 7, 1408-1414, 2014.1592 (2014).

22. Samuels JS, Holland L, López M, et al. Prostaglandin E2 and IL-23 interconnects STAT3 and RoR $\gamma$ pathways to initiate Th17 CD4+ Tcell development during rheumatoid arthritis. Inflamm Res. 67(7):589596(2018).

23. Savtekin G, Sehirli AO. Rheumatoid arthritis in temporo-mandibular joint: A review. Niger J Clin Pract. 21(10):1243-1246(2018).

24. Saw TY, Malik NA, Lim KP, et al. Oral Supplementation of Tocotrienol-Rich Fraction Alleviates Severity of Ulcerative Colitis in Mice. J Nutr Sci Vitaminol (Tokyo). 65(4):318-327 (2019).

25. Scott DL, Wolfe F, Huizinga TW. Rheumatoid arthritis. Lancet. 376(9746):1094-1108(2010).

26. Scott, D. L., Wolfe, F. \& Huizinga, T. W. Rheumatoid arthritis. Lancet (London, England) 376, 1094-1108 (2010).

27. Sproston, N. R. \& Ashworth, J. J. Role of C-Reactive Protein at Sites of Inflammation and Infection. Frontiers in immunology 9, 754 (2018). 
28. Szymańska R, Nowicka B, Kruk J. Vitamin E - Occurrence, Biosynthesis by Plants and Functions in Human Nutrition. Mini Rev Med Chem. 17(12):1039-1052 (2017).

29. Thakur S, Riyaz B, Patil A, Kaur A, Kapoor B, Mishra V. Novel drug delivery systems for NSAIDs in management of rheumatoid arthritis: An overview. Biomed Pharmacother. 106:1011-1023 (2018).

30. Wang W, Zhou H, Liu L. Side effects of methotrexate therapy for rheumatoid arthritis: A systematic review. Eur J Med Chem. 158:502516 (2018).

31. Wei, S. T., Sun, Y. H., Zong, S. H. \& Xiang, Y. B. Serum Levels of IL-6 and TNF-alpha May Correlate with Activity and Severity of Rheumatoid Arthritis. Medical science monitor: international medical journal of experimental and clinical research 21, 4030-4038 (2015).

32. Williams AM, Ladva CN, Leon JS, et al. Changes in micronutrient and inflammation serum biomarker concentrations after a norovirus human challenge. Am J Clin Nutr. 110(6):1456-1464 (2019).

33. Zaida Zainal, Z. S. \& Sitti Rahma, A. H. Composition Comprising Of Palm $\gamma$-Tocotrienol for Treatment of Arthritis.WO2014/133379A (2014). 